University of Nebraska - Lincoln

DigitalCommons@University of Nebraska - Lincoln

USDA National Wildlife Research Center - Staff Publications
U.S. Department of Agriculture: Animal and Plant Health Inspection Service

January 2006

\title{
Liquid chromatography-electrospray ionization mass spectrometry for direct identification and quantification of iophenoxic acid in serum
}

\author{
Melinda C. Wiles \\ Texas Veterinary Medical Diagnostic Laboratory, mwiles@tvmdl.tamu.edu \\ Tyler A. Campbell \\ USDA-APHIS, NWRC, Kingsville, TX Field Station, tcampbell@eastfoundation.net
}

Follow this and additional works at: https://digitalcommons.unl.edu/icwdm_usdanwrc

Part of the Environmental Sciences Commons

Wiles, Melinda C. and Campbell, Tyler A., "Liquid chromatography-electrospray ionization mass spectrometry for direct identification and quantification of iophenoxic acid in serum" (2006). USDA National Wildlife Research Center - Staff Publications. 8.

https://digitalcommons.unl.edu/icwdm_usdanwrc/8

This Article is brought to you for free and open access by the U.S. Department of Agriculture: Animal and Plant Health Inspection Service at DigitalCommons@University of Nebraska - Lincoln. It has been accepted for inclusion in USDA National Wildlife Research Center - Staff Publications by an authorized administrator of DigitalCommons@University of Nebraska - Lincoln. 


\title{
Liquid chromatography-electrospray ionization mass spectrometry for direct identification and quantification of iophenoxic acid in serum
}

\author{
Melinda C. Wiles ${ }^{\mathrm{a}, *}$, Tyler A. Campbell ${ }^{\mathrm{b}}$ \\ ${ }^{a}$ Texas Veterinary Medical Diagnostic Laboratory, P.O. Drawer 3040, College Station, TX 77841, United States \\ ${ }^{\mathrm{b}}$ USDA-APHIS-WS, National Wildlife Research Center, Texas A\&M University-Kingsville, MSC 218, \\ 700 University Blvd., Kingsville, TX 78363, United States \\ Received 16 September 2005; accepted 9 December 2005 \\ Available online 18 January 2006
}

\begin{abstract}
A liquid chromatographic-electrospray ionization mass spectrometric technique was developed for direct quantitation of iophenoxic acid (IA) in serum. IA was spiked into canine, feline, bovine, equine, and porcine sera, extracted, and quantified using negative ion monitoring following chromatographic separation on a Luna C18(2) $3 \mu \mathrm{m}(100 \mathrm{~mm} \times 2.1 \mathrm{~mm})$ reversed-phase column. The limit of detection was $25 \mathrm{ng} / \mathrm{mL}$ and the limit of quantification was $50 \mathrm{ng} / \mathrm{mL}$. Inter- and intra-assay accuracy (86-113\% and 87-115\%, respectively) and precision (1.8-7.7\%) were calculated. Analysis of serum collected from feral pigs, raccoons, and opossums following ingestion of IA-marked baits confirmed the appropriateness of this method for bait acceptance studies.
\end{abstract}

(c) 2005 Elsevier B.V. All rights reserved.

Keywords: LC-MS; Negative ion monitoring; Electrospray ionization; Iophenoxic acid; Serum; Bait acceptance

\section{Introduction}

A major hindrance to disease control, specifically the protection of human health and prevention of economic losses resulting from zoonotic transmission, is the utilization of efficient and cost-effective vaccination programs. For example, in many countries large segments of the dog population, a major contributor to human rabies cases, are not accessible by traditional vaccination routes [1]. However, oral vaccination of free-roaming dogs and other wildlife offers a cheap and efficient alternative in only a fraction of the time that is required to catch, constrain, and vaccinate by the traditional parenteral route. Edible baits containing physiological marking agents have been successfully used to vaccinate coyotes [2], raccoons [3], and dogs [4] against the spread of rabies and to vaccinate prairie dogs for plague [5]. Once the baits are consumed the marking agents may then be measured in the blood of live animals to determine the efficiency of vaccine ingestion rather than attempting to locate animals that have died of the disease.

\footnotetext{
* Corresponding author. Tel.: +1 9798453414.

E-mail address: mwiles@tvmdl.tamu.edu (M.C. Wiles).
}

Iophenoxic acid ( $\alpha$-ethyl-3-hydroxy-2,4,6-triiodohydrocinnamic acid; IA) is an organic, iodine-containing compound that has been used as a marker in so called "bait acceptance" studies (Fig. 1). These studies have been conducted in a variety of animal species including carnivores, such as raccoons, foxes, dogs, coyotes and cats [6-11], herbivores, such as goats, rabbits and deer [12-16] and omnivores, such as pigs [17]. The compound is effective because it exhibits low toxicity while strongly binding to albumin over an extended length of time $[18,19]$. In addition, while the extent of binding is both dose- and speciesdependent [11], IA may be used to determine the amount of bait consumed $[10,12,16]$. However, the compound persists for only short durations in marsupials, including opossums [11] or swamp wallabies [20], and birds [6].

Previous methods of identification and quantification primarily involve indirect inference by measuring plasma or serum iodine, which show gross and prolonged elevation as a function of IA concentration $[6,10,13,21]$. A more recent method describes direct determination of the compound using HPLC followed by UV detection [22]. Although this method has been shown to be quite sensitive, it requires an injection volume of $300 \mu \mathrm{L}$ to achieve this sensitivity. The objective of the present study was to examine the use of LC-MS as an 


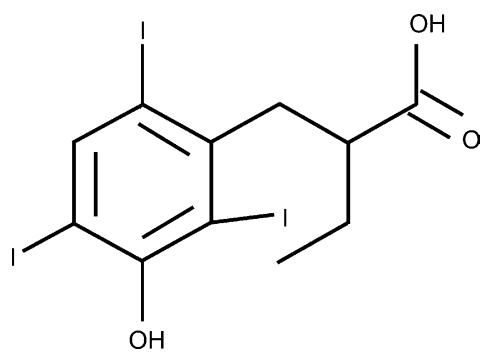

Fig. 1. Chemical structure of iophenoxic acid $\left(\mathrm{C}_{11} \mathrm{H}_{11} \mathrm{I}_{3} \mathrm{O}_{3}\right.$; formula weight $=571.92$ ).

alternative method. Following a simple extraction procedure, liquid chromatography coupled to electrospray ionization mass spectroscopy in the negative ion mode allows for unequivocal identification and reliable quantification of IA in serum.

\section{Materials and methods}

\subsection{Chemical and reagents}

Iophenoxic acid ( $\alpha$-ethyl-3-hydroxy-2,4,6-triiodohydrocinnamic acid; $98 \%$; IA) was purchased from Aldrich (Milwaukee, WI). ACS grade methanol and GR grade sulfuric acid were purchased from EM Science (Gibbstown, NJ), glacial acetic acid was purchased from J.T. Baker (Phillipsburg, NJ), and sodium tungstate dihydrate was received from Sigma (St. Louis, $\mathrm{MO})$. Water was deionized in the laboratory $\left(\mathrm{dH}_{2} \mathrm{O}\right)$. Solvents used as LC-MS mobile phases were HPLC grade acetonitrile (EM Science, Gibbstown, NJ) and ultrapure water $\left(\mathrm{UP}-\mathrm{H}_{2} \mathrm{O}\right.$, Burdick and Jackson, Muskegon, MI). Liquid nitrogen was obtained from Praxair (Bryan, TX).

\subsection{Sample collection}

All bench work was conducted using feline (Felis domesticus), canine (Canis familiaris), bovine (Bos taurus), equine (Equis caballas), and porcine (Sus scrofa) serum that was collected from specimens submitted to the Texas Veterinary Medical Diagnostic Laboratory (TVMDL) for unrelated testing procedures and pooled. Aliquots $(400 \mu \mathrm{L})$ were spiked with IA immediately prior to each extraction.

\subsection{Preparation of standard solutions for spiking}

A stock solution of $5000 \mu \mathrm{g} / \mathrm{mL}$ IA in methanol was serially diluted in order to obtain working solutions ranging in concentration from 12.5 to $5000 \mu \mathrm{g} / \mathrm{mL}$ IA. These solutions were then spiked into serum in $10 \mu \mathrm{L}$ amounts in order to obtain concentrations ranging from 25 to $5000 \mathrm{ng} / \mathrm{mL}$ in the final $5 \mathrm{~mL}$ extract.

\subsection{Sample preparation: field analysis}

Field experiments were conducted on 1700 ha of private land in Duval County, TX. PIGOUT ${ }^{\circledR}$ Feral Pig Baits (Prototype 2) (Animal Control Technologies, Somerton, Victoria, Australia) were spiked with IA by drilling a $0.6 \mathrm{~cm}$ hole into the center of the bait. The marker was injected at $22.7 \mathrm{mg} / \mathrm{bait}$, a quantity known to mark feral pig serum [17]. On day 1, 1170 IA marked baits were placed on the property on transects $(n=18)$ oriented on a $273^{\circ}$ azimuth and spaced at $260 \mathrm{~m}$ intervals. On each transect, baits were placed every $65 \mathrm{~m}$ resulting in an approximate density of 68 baits $/ \mathrm{km}^{2}$.

Fifteen corral style and nine box style feral pig traps and 20 mesomammal live traps (Tomahawk Live Trap, Tomahawk, WI) were used to capture animals. Traps were placed in shaded areas, to prevent trapped animals from over-heating, at an approximate density of 1.4 traps $/ \mathrm{km}^{2}$. On day 6 the traps were baited and set and then checked from days 7 to 15. Captured feral pigs (S. scrofa), raccoons (Procyon lotor), and opossums (Didelphis virginiana) were sexed, aged and bled. Serum was separated from the collected blood, placed in labeled tubes and frozen at $-20^{\circ} \mathrm{C}$ prior to analysis. Non-target animals were released upon discovery.

\subsection{Serum extraction}

The extraction protocol has previously been published [22]. Briefly, $0.4 \mathrm{~mL}$ serum was added to a polypropylene Falcon test tube (Becton Dickinson, Franklin Lakes, NJ). For spiked solutions, $10 \mu \mathrm{L}$ of the appropriate concentrated IA standard was then added using a $10 \mu \mathrm{L}$ Hamilton syringe. To all standards and samples, $0.8 \mathrm{~mL}$ of $0.33 \mathrm{M}$ sulfuric acid, $0.8 \mathrm{~mL}$ of a sodium tungstate dihydrate solution $(10 \%, w / v$ in water $)$, and $3 \mathrm{~mL}$ methanol were added. The solutions were vortexed briefly to mix, allowed to stand at room temperature (ca. $22^{\circ} \mathrm{C}$ ) for $15 \mathrm{~min}$, and then centrifuged at $1800 \times g, 25^{\circ} \mathrm{C}$, for $20 \mathrm{~min}$ using an Allegra $^{\mathrm{TM}}$ 6R Centrifuge (Beckman Coulter, Fullerton, CA). The supernatant was transferred to a glass, graduated $10 \mathrm{~mL}$ conical tube and the volume was adjusted to $5 \mathrm{~mL}$ with a 3:2 solution of methanol: $\mathrm{dH}_{2} \mathrm{O}$. A portion of this extract was then transferred to a glass, crimp-cap, $2 \mathrm{~mL}$ autosampler vial (Agilent, Palo Alto, CA) for LC-MS analysis.

\subsection{LC-MS analysis}

To qualitatively and quantitatively determine iophenoxic acid in serum samples, $20 \mu \mathrm{L}$ were injected onto a Luna C18(2) column $(100 \AA ̊ ., 3 \mu \mathrm{m}, 100 \mathrm{~mm} \times 2.1 \mathrm{~mm}$, Phenomenex, Torrance, CA) with a $4.0 \mathrm{~mm} \times 2.0 \mathrm{~mm}$ SecurityGuard guard column (Phenomenex, Torrance, CA) using a Finnigan Surveyor autosampler and a Finnigan Surveyor MS pump (Finnigan, West Palm Beach, FL). The samples were separated by gradient elution with $\mathrm{UP}-\mathrm{H}_{2} \mathrm{O}$ containing $0.1 \%$ acetic acid (solvent $\mathrm{A}$ ) and HPLC-grade acetonitrile containing $0.2 \%$ acetic acid (solvent B) under the following conditions: $50 \% \mathrm{~A}$ isocratic for $0.5 \mathrm{~min}$, linear to $5 \%$ A within $7 \mathrm{~min}$, isocratic at $5 \%$ A for 5 min, linear to $50 \%$ A within $2 \mathrm{~min}$, isocratic at $50 \%$ A to re-establish original conditions. The flow rate was $300 \mu \mathrm{L} / \mathrm{min}$. The LC system was coupled directly to a Finnigan LCQ Deca XP $\mathrm{XAX}^{\mathrm{MA}}$ ion trap mass spectrometer (Finnigan, West Palm Beach, FL) equipped with an electrospray ionization (ESI) probe.

Analytes were detected using negative ion monitoring at a capillary temperature of $300{ }^{\circ} \mathrm{C}$, spray voltage of $4.5 \mathrm{kV}$, capil- 
lary voltage of $-16 \mathrm{~V}$, and tube lens offset of $-35 \mathrm{~V}$. Spectral data were recorded with $\mathrm{N}_{2}$ as collision gas (sheath gas of 40 arbitrary units and auxiliary/sweep gas of 10 arbitrary units). Detection and quantification were performed in SIM mode $(\mathrm{m} / \mathrm{z}$ 571) due to enhanced linearity at low concentrations. Detection was confirmed using $\mathrm{m} / \mathrm{z} 443$ following MS/MS of $\mathrm{m} / \mathrm{z}$ 571. The MS/MS collision energy was set to $40 \%$. For purposes of presentation, data for figures in this paper was collected using dual analysis in full SCAN ( $\mathrm{m} / z, 200-900)$ and MS/MS modes. Chromatograms represent isolation of $\mathrm{m} / \mathrm{z} 571$ from the full scan data, however, the associated MS data presented for that specific retention time is full scan $(\mathrm{m} / \mathrm{z}, 200-900)$ at that specific retention time. MS/MS data was collected and is presented as full scan data. Data were collected and processed using Xcalibur 1.4 software (Finnigan, West Palm Beach, FL).

\subsection{Analyte quantitation}

Quantitation of analytes in both bench and field samples was based on calibration curves obtained daily from spiked and extracted serum. Quantitation of the extraction product was performed using SIM data $(m / z, 571)$. Calibrations curves were obtained by plotting the extracted ion current peak area of the spiked serum extract versus expected concentration using weighted linear regression. The mean correlation coefficient, expressed as $R^{2}$, was used to judge linearity. The limit of quantitation (LOQ) was defined as the concentration routinely having intra-day variability of less than $20 \%$ and mean accuracy of back calculations that was within $20 \%$ of the expected concentration. Accuracy was defined as the amount measured divided by the amount claimed. Inter-assay accuracy was calculated from pooled serum that was separated into four independent aliquots. Each aliquot was spiked with IA in order to obtain a final concentration of 25, 50, 100, 250, 500, 1000, or $5000 \mathrm{ng} / \mathrm{mL}$ and extracted. Values were reported as a range for four sets of standards extracted independently on four separate days. Intra-assay accuracy was reported as the range of values obtained from one of these aliquots that was injected four separate times. Precision values were expressed as the relative standard deviation of 11 consecutive injections of four different concentrations of IA that was spiked into serum and extracted for final expected values of 100, 250, 500, and $1000 \mathrm{ng} / \mathrm{mL}$.

\section{Results and discussion}

\subsection{Sample extraction and detection}

To ensure adequate recovery of serum-bound IA for LC-MS determination an extraction method designed to completely dissociate the compound from serum albumin was selected [22]. Previous research has shown this tungstate- $\mathrm{H}_{2} \mathrm{SO}_{4}$ procedure to be extremely effective for protein precipitation [23]. However, unlike previous studies which utilized UV to detect and quantitate the compound, LC-MS was selected as a more definitive method of analysis. The limits of detection and quantification ranged from 500 to $1000 \mathrm{ng} / \mathrm{mL}$ using electrospray ionization in the positive ion mode $((+) E S I)$ and atmospheric pressure chemical ionization (APCI) did not produce either protonated or sodiated adducts that are characteristic of LC-MS. Therefore, these modes of analysis were not pursued. In contrast, electrospray ionization in the negative ion mode $((-)$ ESI) exhibited adequate sensitivity and reliability for the purpose of the study. The gradient system used in our methodology gave rise to sharp, nearly symmetrical peaks. A six-point quantification curve of the concentration of IA versus peak area was constructed for quantitation of concentrations as low as $50 \mathrm{ng} / \mathrm{mL}$ in serum and the correlation coefficients were routinely greater than 0.9900. It may be unwise to examine levels lower than these, at least in the case of bait acceptance studies, as previous research using serum iodine as a measure of IA has reported background levels in some species to range between 20 and $50 \mathrm{ng} / \mathrm{mL}$ with some as high as $80 \mathrm{ng} / \mathrm{mL}[6,7]$. However, it is unknown whether these background iodine levels are purely the result of previous IA exposure. In addition, in many species IA is strongly bound to serum albumin over an extended period of time. For example, one study conducted in beagle dogs that were fed $20 \mathrm{mg}$ tablets showed serum iodine present at levels at least double that of baseline values after 34-52 weeks [7].

\subsection{Analyte identification}

Using this LC-MS technique, acidic compounds such as IA form a deprotonated molecule $[\mathrm{M}-\mathrm{H}]^{-}$that can be analyzed in the negative ion mode as the quasi-molecular ion peak at $m / z \mathrm{M}-1$. Typical chromatograms and associated mass spectral data are presented for extracts of IA dissolved in methanol and spiked into water (Fig. 2a), bovine (B. taurus) (Fig. 3a), equine (E. caballas) (Fig. 4a), feline (F. domesticus) (Fig. 5a), canine (C. familiaris) (Fig. 6a), and porcine serum (Fig. 7a). Solvent (Fig. 2b) and serum blanks were also analyzed (Figs. 3b-7b). The mass spectra all contain $\mathrm{m} / \mathrm{z}, 571$ as the characteristic product ion. MS/MS fragmentation of this ion produced a characteristic product ion at $\mathrm{m} / \mathrm{z} 443$ which was used for IA confirmation. However, under the conditions used in this research SIM data exhibited greater linearity over a broader range of concentrations and was therefore used for IA quantification. MS/MS data frequently exhibited appropriate linearity and accuracy $\left(R^{2}=0.9991-09998\right.$; accuracy $\left.=98-102 \%\right)$ between 250 and $1000 \mathrm{ng} / \mathrm{mL}$ but exhibited low responses for greater concentrations and higher responses for lesser IA concentrations outside of this range. Thus, this method was shown to be effective for identification but not as robust over a wider range of concentrations. No interferences were observed in serum or method blanks.

\subsection{Stability}

Linearity was observed between the intensity of $\mathrm{m} / \mathrm{z}, 571$ and the concentration of IA in serum extracts over an extended period of time. Sample extracts containing concentrations of IA ranging from 50 to $5000 \mathrm{ng} / \mathrm{mL}$ exhibited linearity with respect to the 

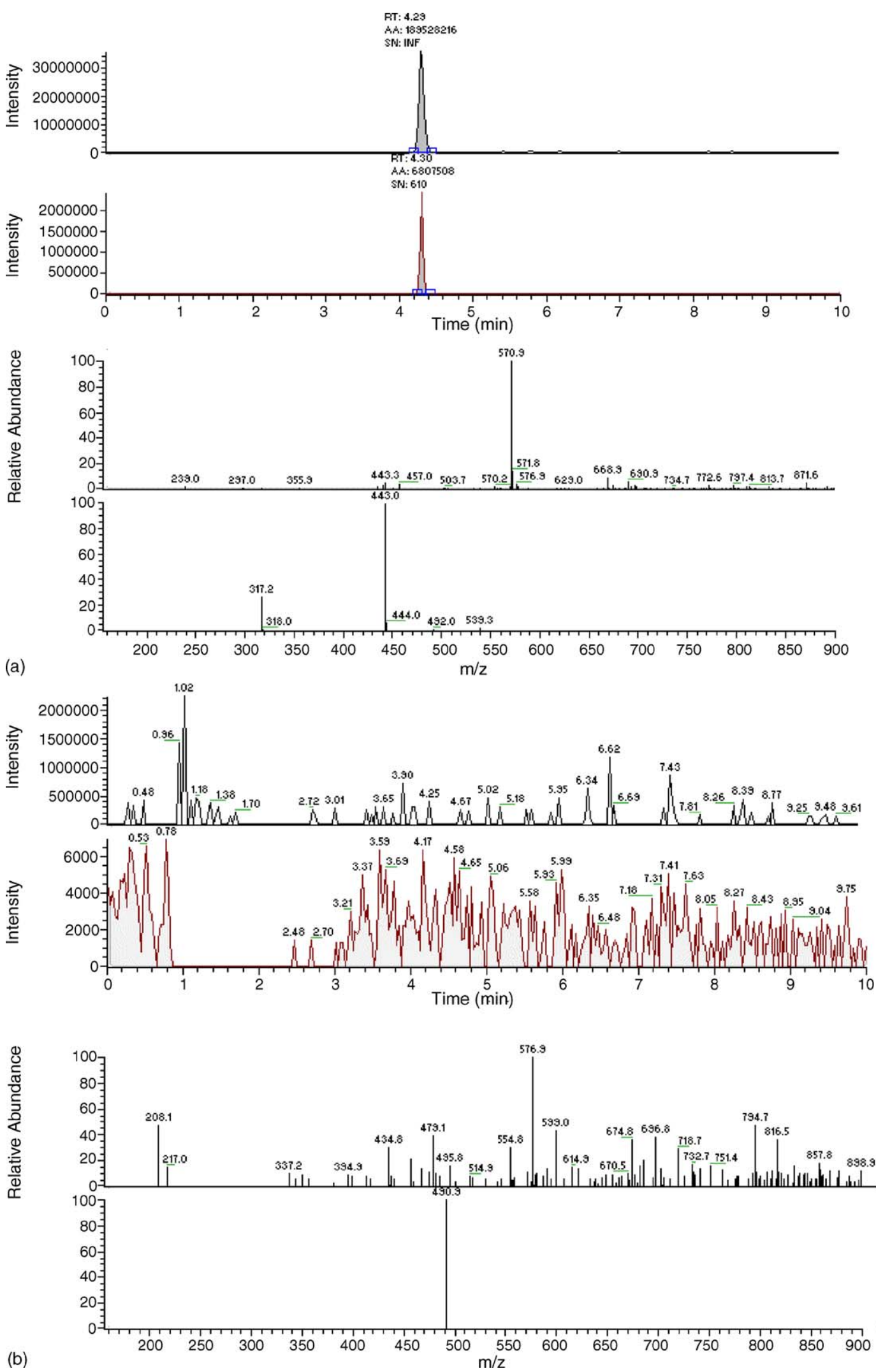

Fig. 2. Typical chromatogram and mass spectra of IA standard spiked into water for a final concentration of $500 \mathrm{ng} / \mathrm{mL}$ in the extract (a) as compared to an unspiked water extract (method blank) (b). The data shows $[\mathrm{M}-\mathrm{H}]^{-}(\mathrm{m} / \mathrm{z}$ 571) at 4.29 min isolated from full scan data and the corresponding full scan mass spectral data for this peak (upper chromatogram and upper mass spectra) as well as the peak resulting from MS/MS of the $[\mathrm{M}-\mathrm{H}]^{-}$peak at $4.30 \mathrm{~min}$ and its corresponding fragmentation pattern. The MS/MS data was used to confirm the detection of IA (lower chromatogram and mass spectrum). Data was obtained using dual analysis in full scan (200-900 amu) and MS/MS fragmentation of $\mathrm{m} / \mathrm{z}, 571$ at $40 \%$ normalized collision energy. 

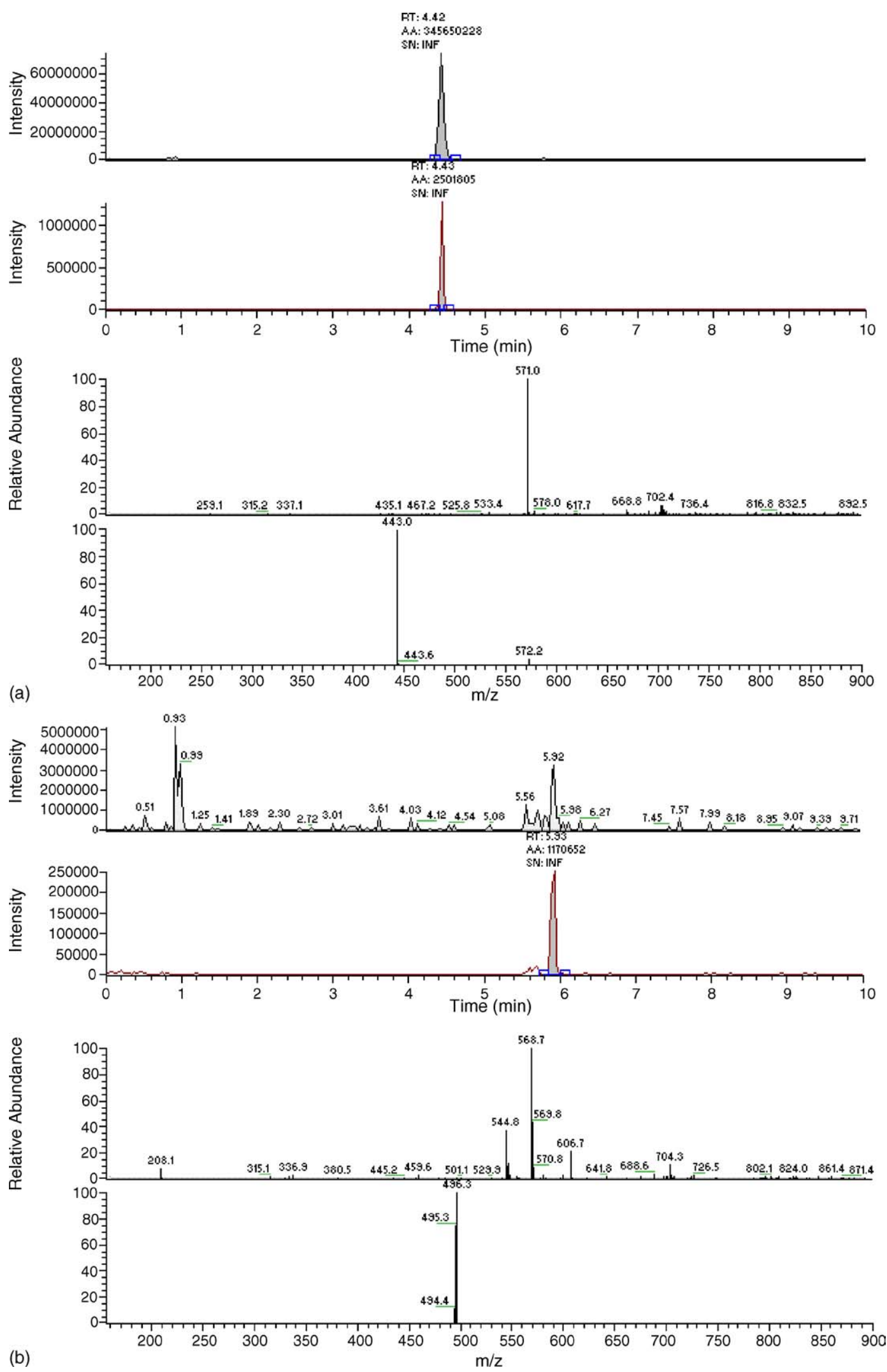

Fig. 3. Typical chromatogram and mass spectra of IA standard spiked into bovine serum for a final concentration of $500 \mathrm{ng} / \mathrm{mL}$ in the extract (a) as compared to an unspiked bovine serum extract (b). The data shows $[\mathrm{M}-\mathrm{H}]^{-}(\mathrm{m} / \mathrm{z}$ 571) at $4.42 \mathrm{~min}$ isolated from full scan data and the corresponding full scan mass spectral data for this peak (upper chromatogram and upper mass spectra) as well as the peak resulting from MS/MS of the $[\mathrm{M}-\mathrm{H}]^{-}$peak at 4.43 min and its corresponding fragmentation pattern. The MS/MS data was used to confirm the detection of IA (lower chromatogram and mass spectrum). Data was obtained using dual analysis in full scan (200-900 amu) and MS/MS fragmentation of $m / z 571$ at $40 \%$ normalized collision energy. 

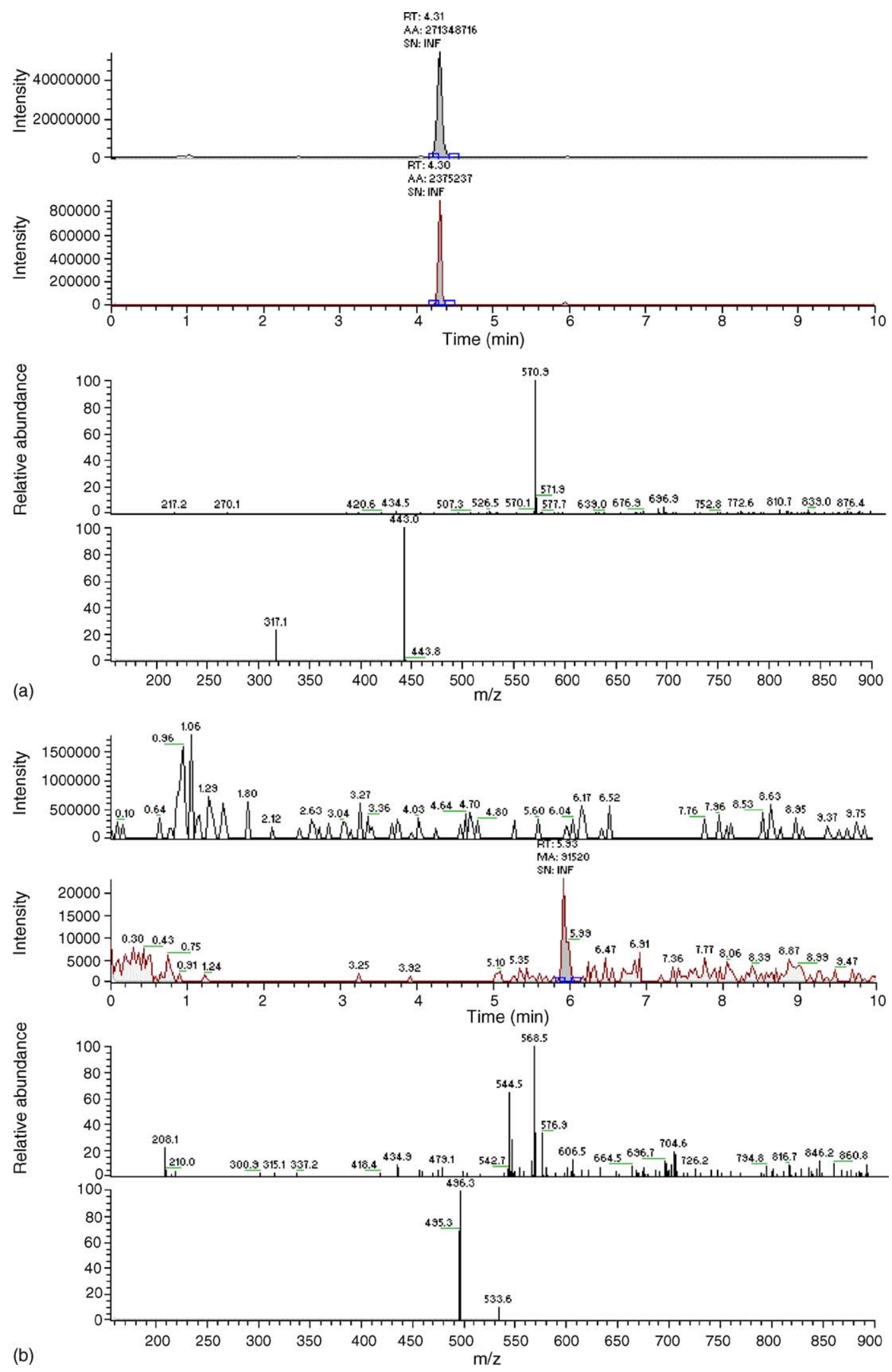

Fig. 4. Typical chromatogram and mass spectra of IA standard spiked into equine serum for a final concentration of $500 \mathrm{ng} / \mathrm{mL}$ in the extract (a) as compared to an unspiked equine serum extract (b). The data shows $[\mathrm{M}-\mathrm{H}]^{-}(\mathrm{m} / \mathrm{z}, 571)$ at $4.31 \mathrm{~min}$ isolated from full scan data and the corresponding full scan mass spectral data for this peak (upper chromatogram and upper mass spectra) as well as the peak resulting from MS/MS of the $[\mathrm{M}-\mathrm{H}]^{-}$peak at 4.30 min and its corresponding fragmentation pattern. The MS/MS data was used to confirm the detection of IA (lower chromatogram and mass spectrum). Data was obtained using dual analysis in full scan (200-900 amu) and MS/MS fragmentation of $\mathrm{m} / \mathrm{z}, 571$ at $40 \%$ normalized collision energy. 

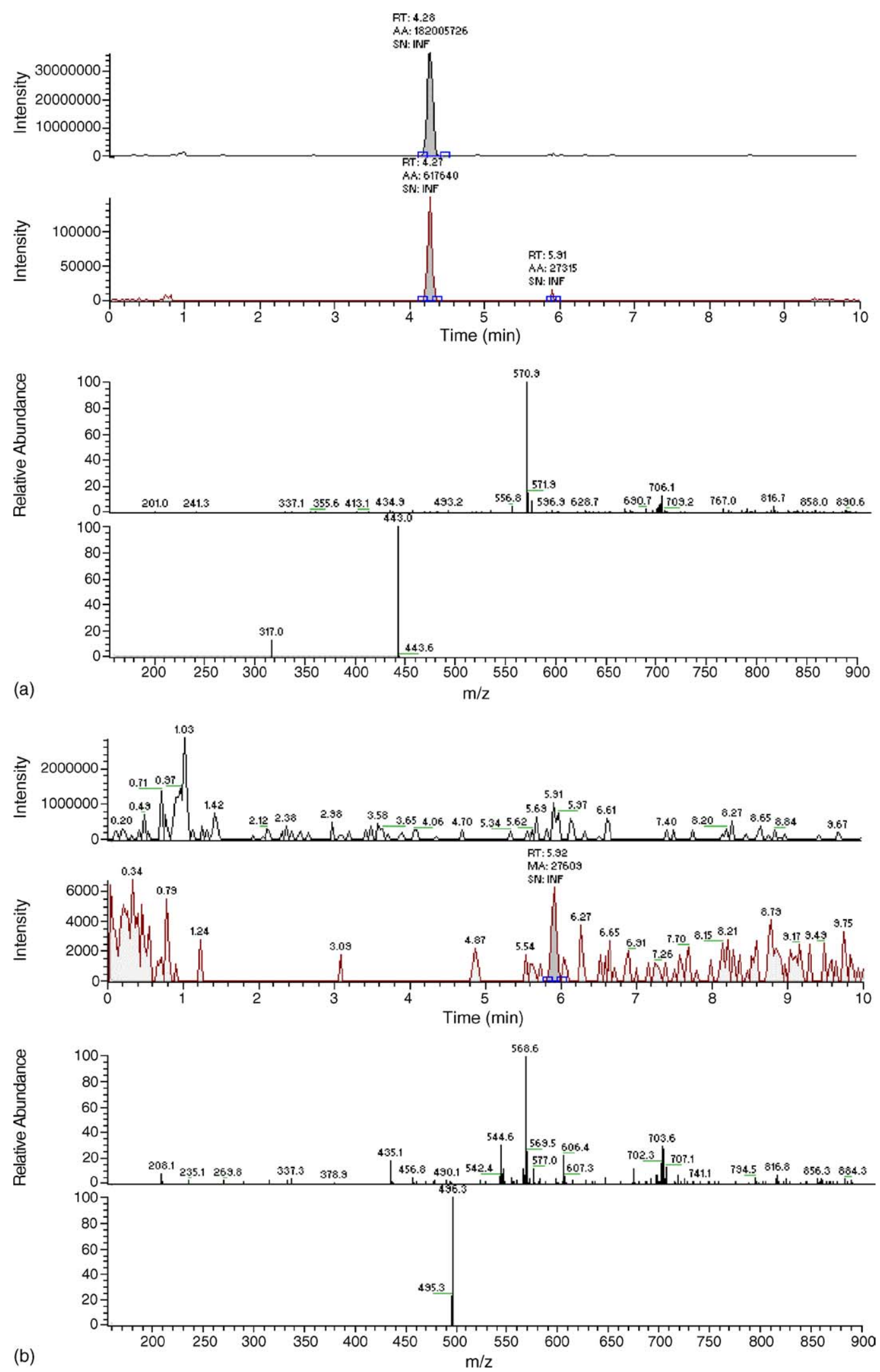

Fig. 5. Typical chromatogram and mass spectra of IA standard spiked into feline serum for a final concentration of $500 \mathrm{ng} / \mathrm{mL}$ in the extract (a) as compared to an unspiked feline serum extract (b). The data shows $[\mathrm{M}-\mathrm{H}]^{-}(\mathrm{m} / \mathrm{z}, 571)$ at $4.28 \mathrm{~min}$ isolated from full scan data and the corresponding full scan mass spectral data for this peak (upper chromatogram and upper mass spectra) as well as the peak resulting from MS/MS of the $[\mathrm{M}-\mathrm{H}]^{-}$peak at 4.27 min and its corresponding fragmentation pattern. The MS/MS data was used to confirm the detection of IA (lower chromatogram and mass spectrum). Data was obtained using dual analysis in full scan (200-900 amu) and MS/MS fragmentation of $m / z 571$ at $40 \%$ normalized collision energy. 

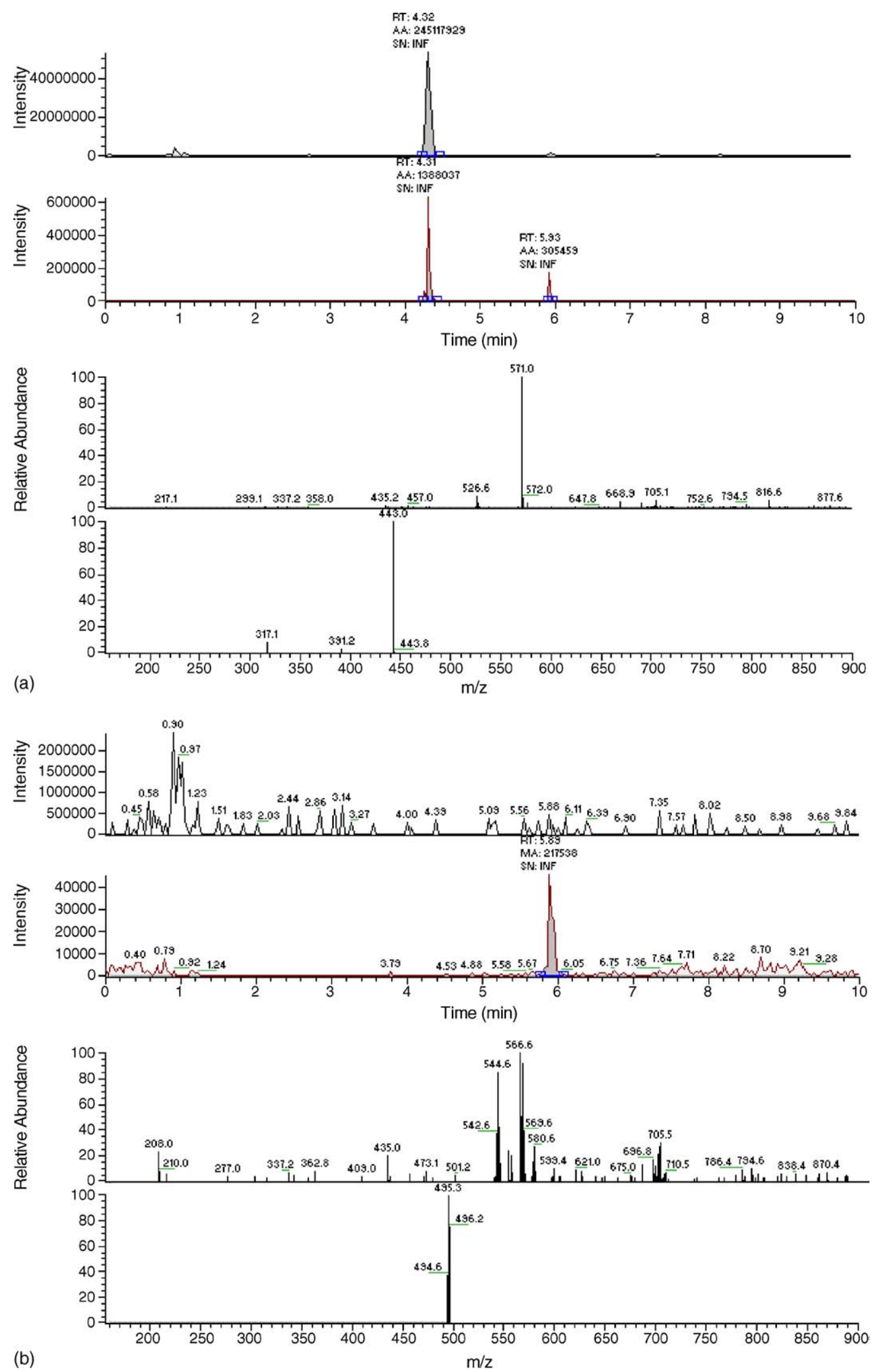

Fig. 6. Typical chromatogram and mass spectra of IA standard spiked into canine serum for a final concentration of $500 \mathrm{ng} / \mathrm{mL}$ in the extract (a) as compared to an unspiked canine serum extract (b). The data shows $[\mathrm{M}-\mathrm{H}]^{-}(\mathrm{m} / \mathrm{z}, 571)$ at $4.32 \mathrm{~min}$ isolated from full scan data and the corresponding full scan mass spectral data for this peak (upper chromatogram and upper mass spectra) as well as the peak resulting from MS/MS of the $[\mathrm{M}-\mathrm{H}]^{-}$peak at 4.31 min and its corresponding fragmentation pattern. The MS/MS data was used to confirm the detection of IA (lower chromatogram and mass spectrum). Data was obtained using dual analysis in full scan (200-900 amu) and MS/MS fragmentation of $\mathrm{m} / \mathrm{z}, 571$ at $40 \%$ normalized collision energy. 

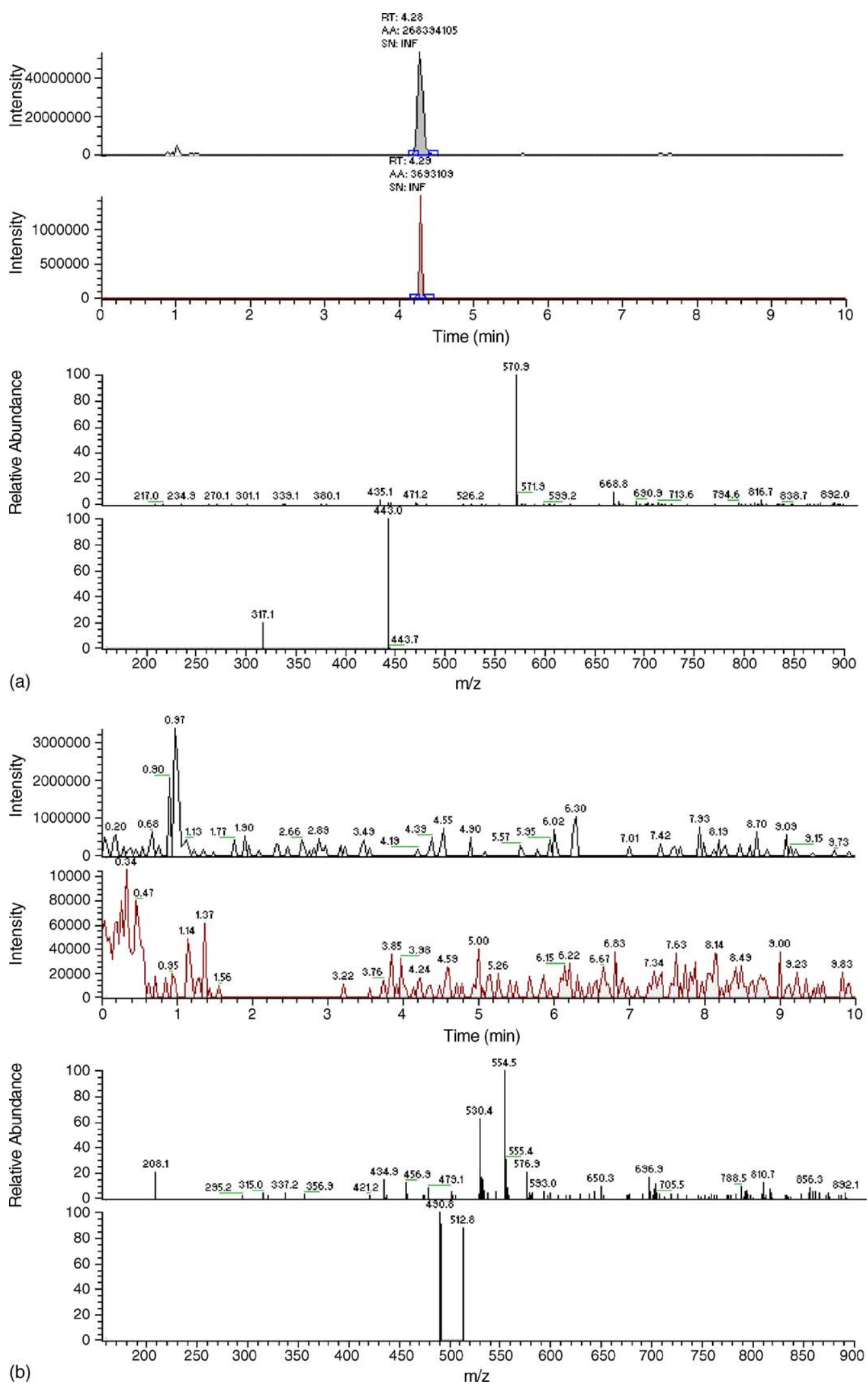

Fig. 7. Typical chromatogram and mass spectra of IA standard spiked into porcine serum for a final concentration of $500 \mathrm{ng} / \mathrm{mL}$ in the extract (a) as compared to an unspiked porcine serum extract (b). The data shows $[\mathrm{M}-\mathrm{H}]^{-}(\mathrm{m} / \mathrm{z}, 571)$ at $4.28 \mathrm{~min}$ isolated from full scan data and the corresponding full scan mass spectral data for this peak (upper chromatogram and upper mass spectra) as well as the peak resulting from MS/MS of the $[\mathrm{M}-\mathrm{H}]^{-}$peak at 4.29 min and its corresponding fragmentation pattern. The MS/MS data was used to confirm the detection of IA (lower chromatogram and mass spectrum). Data was obtained using dual analysis in full scan (200-900 amu) and MS/MS fragmentation of $m / z 571$ at $40 \%$ normalized collision energy. 

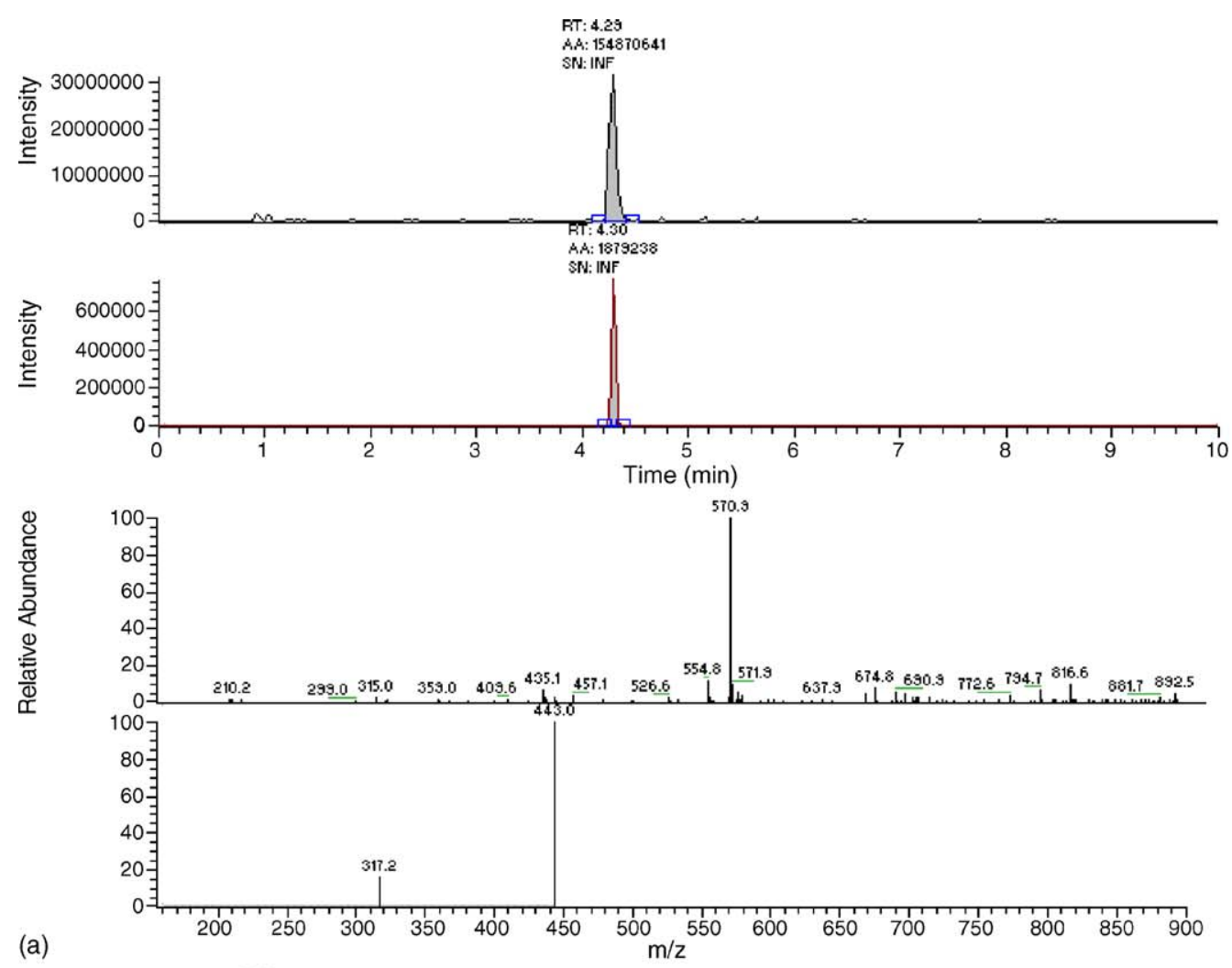

(a)
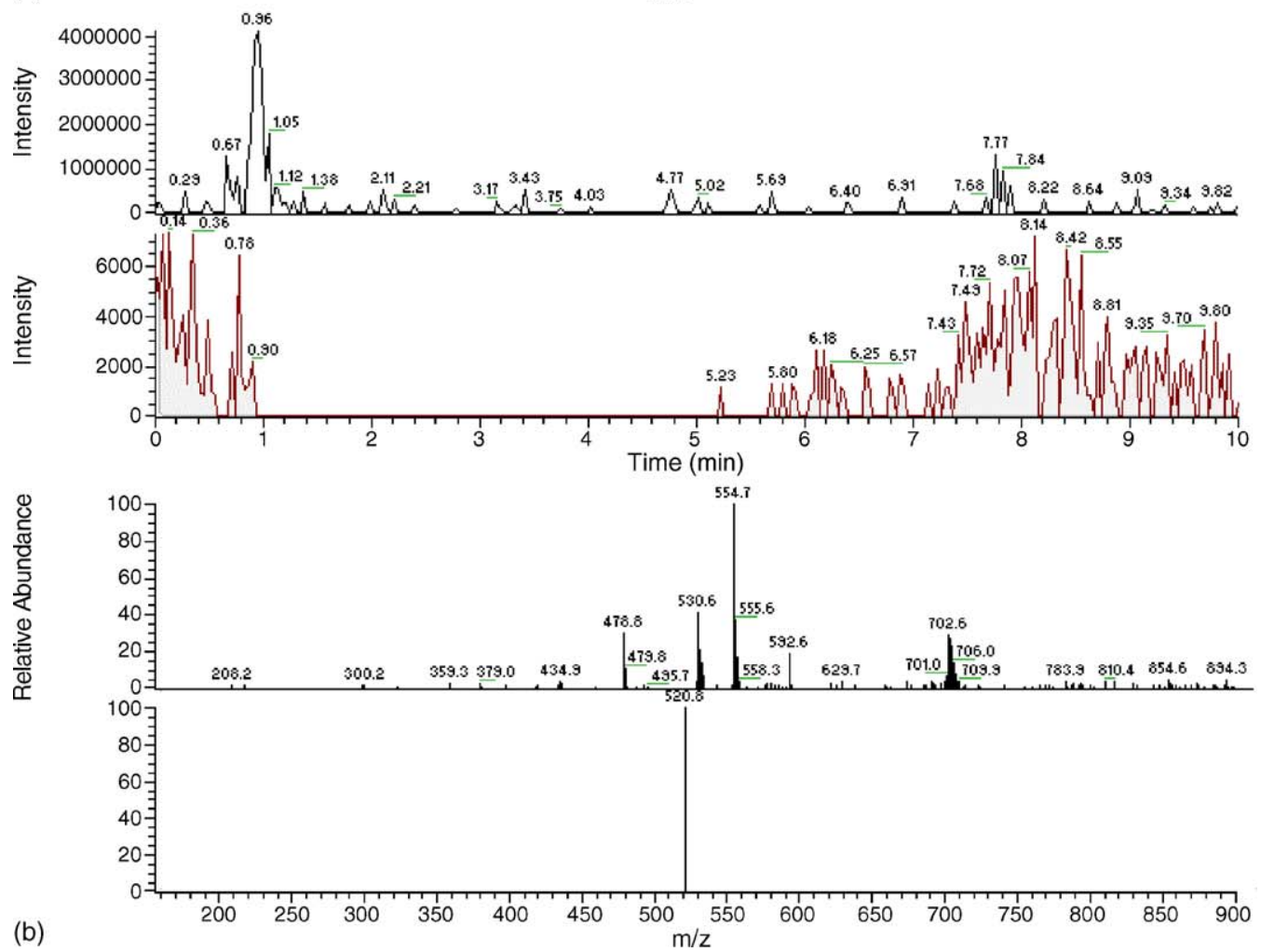

Fig. 8. Typical chromatogram and mass spectra of porcine serum extract following ingestion of IA spiked bait (a) as compared to porcine serum of negative control animals (b). The data shows $[\mathrm{M}-\mathrm{H}]^{-}(\mathrm{m} / \mathrm{z}$ 571) at $4.29 \mathrm{~min}$ isolated from full scan data and the corresponding full scan mass spectral data for this peak (upper chromatogram and upper mass spectra) as well as the peak resulting from MS/MS of the $[\mathrm{M}-\mathrm{H}]^{-}$peak at 4.30 min and its corresponding fragmentation pattern. The MS/MS data was used to confirm the detection of IA (lower chromatogram and mass spectrum). Data was obtained using dual analysis in full scan (200-900 amu) and MS/MS fragmentation of $\mathrm{m} / \mathrm{z} 571$ at $40 \%$ normalized collision energy. 

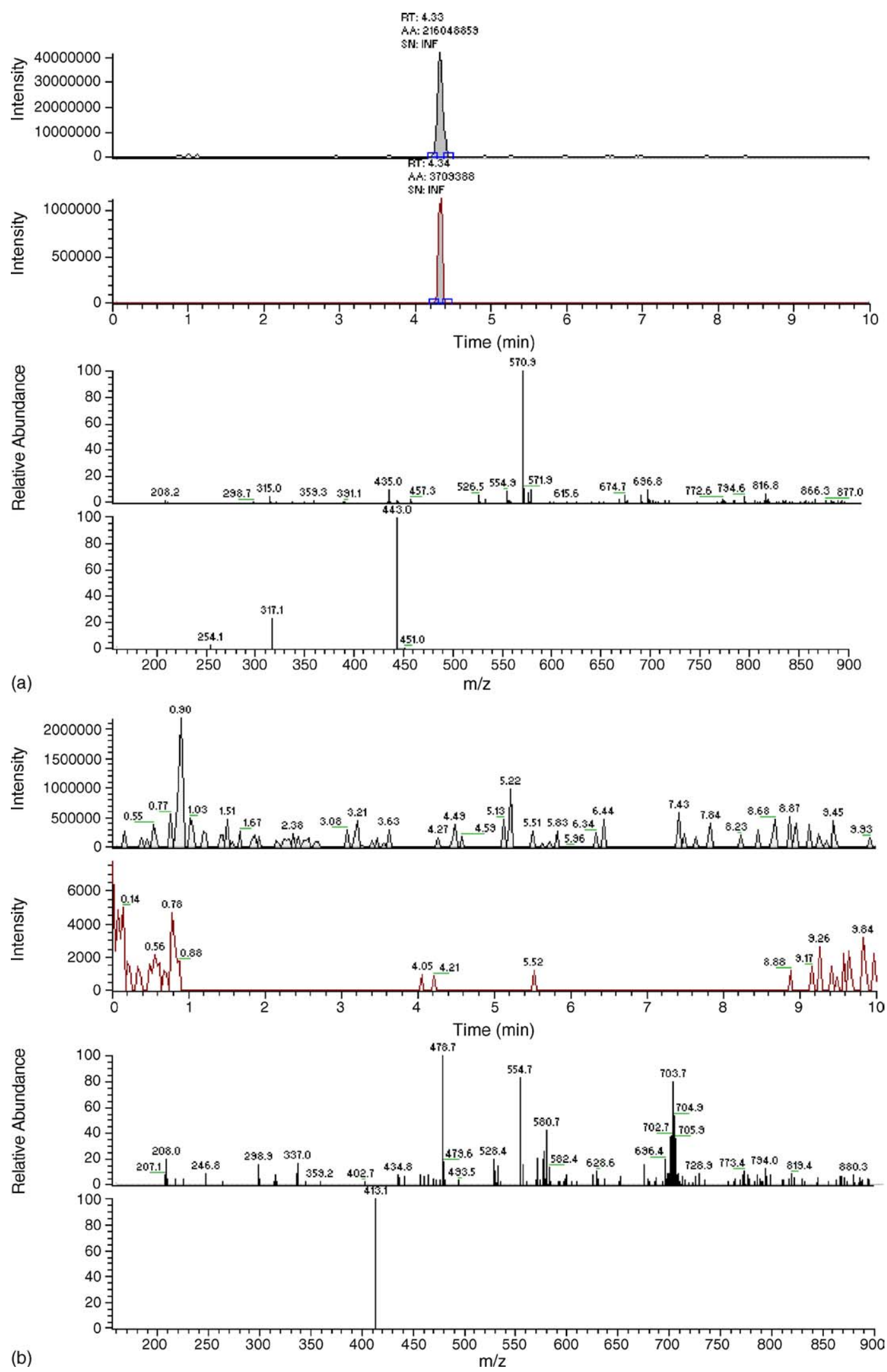

Fig. 9. Typical chromatogram and mass spectra of opossum serum extract following ingestion of IA spiked bait (a) as compared to opossum serum of negative control animals (b). The data shows $[\mathrm{M}-\mathrm{H}]^{-}(\mathrm{m} / \mathrm{z}, 571)$ at $4.33 \mathrm{~min}$ isolated from full scan data and the corresponding full scan mass spectral data for this peak (upper chromatogram and upper mass spectra) as well as the peak resulting from MS/MS of the $[\mathrm{M}-\mathrm{H}]^{-}$peak at 4.34 min and its corresponding fragmentation pattern. The MS/MS data was used to confirm the detection of IA (lower chromatogram and mass spectrum). Data was obtained using dual analysis in full scan (200-900 amu) and MS/MS fragmentation of $\mathrm{m} / \mathrm{z} 571$ at $40 \%$ normalized collision energy. 

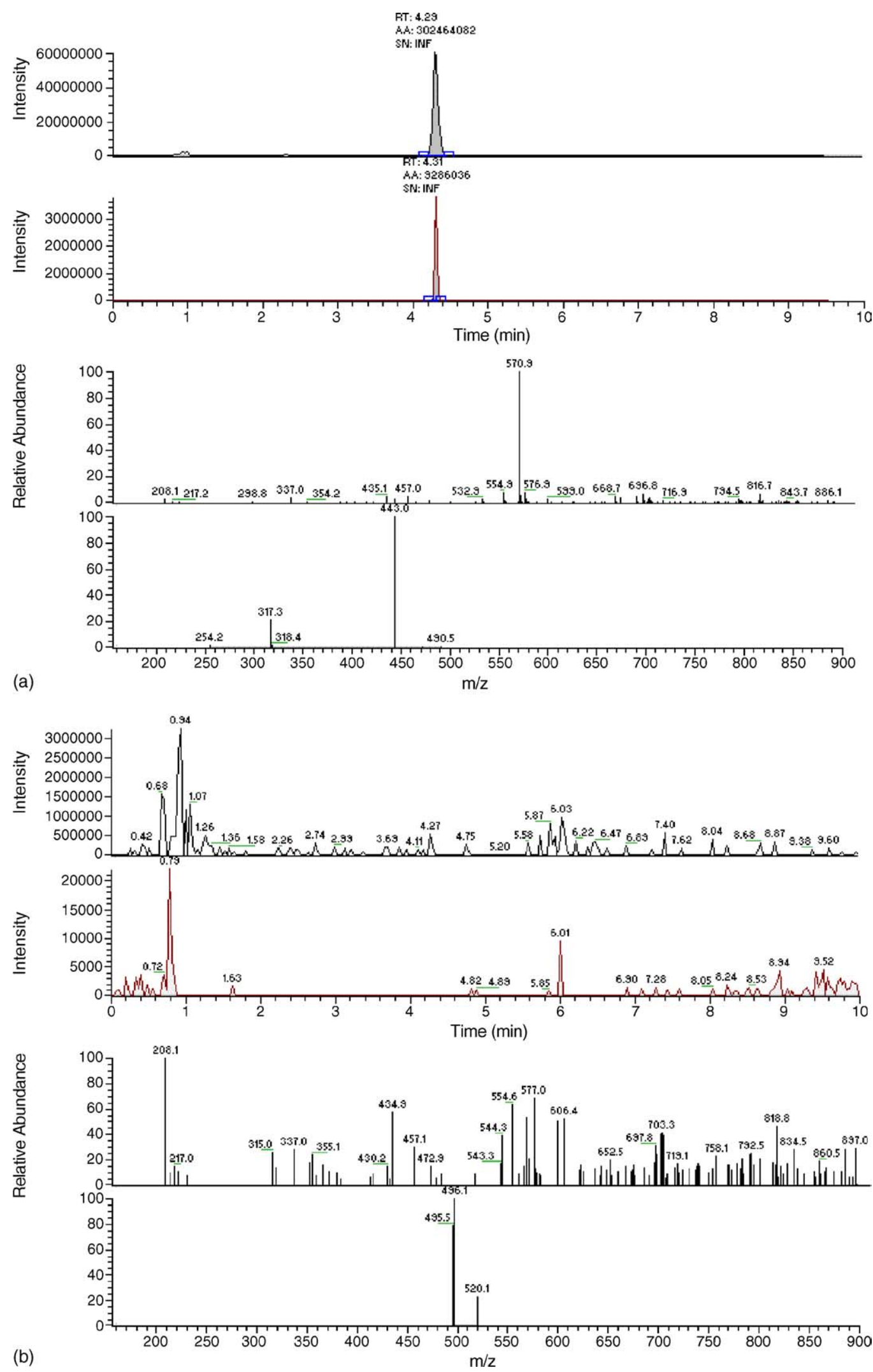

Fig. 10. Typical chromatogram and mass spectra of raccoon serum extract following ingestion of IA spiked bait (a) as compared to raccoon serum of negative control animals (b). The data shows $[\mathrm{M}-\mathrm{H}]^{-}(\mathrm{m} / \mathrm{z}$ 571) at $4.29 \mathrm{~min}$ isolated from full scan data and the corresponding full scan mass spectral data for this peak (upper chromatogram and upper mass spectra) as well as the peak resulting from MS/MS of the $[\mathrm{M}-\mathrm{H}]^{-}$peak at 4.31 min and its corresponding fragmentation pattern. The MS/MS data was used to confirm the detection of IA (lower chromatogram and mass spectrum). Data was obtained using dual analysis in full scan (200-900 amu) and MS/MS fragmentation of $m / z 571$ at $40 \%$ normalized collision energy. 
Table 1

Inter- and intra-assay accuracy and precision of pooled porcine serum samples that were spiked with IA, extracted, and analyzed by (-)ESI/LC-MS

\begin{tabular}{lllllll}
\hline & \multicolumn{6}{l}{ Final concentration of iophenoxic acid in extract $(\mathrm{ng} / \mathrm{mL})$} \\
\cline { 2 - 7 } & 50 & 100 & 250 & 500 & 1000 & 5000 \\
\hline Inter-assay accuracy $(\%)(n=4)$ & $86-101$ & $92-109$ & $97-104$ & $97-113$ & $92-100$ & $99-100$ \\
Intra-assay accuracy $(\%)(n=4)$ & $87-95$ & $103-115$ & $97-110$ & $100-105$ & $94-99$ & $99-100$ \\
Precision $(\%)(n=11)$ & N/A & 7.7 & 3.8 & 5.6 & N/A & 1.8 \\
\hline
\end{tabular}

intensity of $\mathrm{m} / \mathrm{z}, 571$ for at least 2 months when stored at $-20^{\circ} \mathrm{C}$ (data not shown).

\subsection{Accuracy and precision}

Accuracy and precision measurements were performed using porcine serum. Feline, canine, bovine, and equine serum as well as additional samples consisting of spiked water were used to confirm the appropriateness of the method across species. Quantification curves constructed to compare peak areas to known spike concentrations were linear within the range of $50-5000 \mathrm{ng} / \mathrm{mL}$. While adequate for detection, the $25 \mathrm{ng} / \mathrm{mL}$ spike exhibited poor reproducibility as defined by both intra-day variability that was greater than $20 \%$ and mean accuracy of back calculations of expected values that varied by greater than $20 \%$. All other spiked concentrations showed a mean accuracy back calculation of the expected values that varied by no greater than $15 \%$, frequently by no greater than $10 \%$. Thus, $25 \mathrm{ng} / \mathrm{mL}$ was defined as the limit of detection while $50 \mathrm{ng} / \mathrm{mL}$ was selected as the limit of quantification.

Inter-assay accuracy was calculated from pooled porcine serum that was separated into four independent aliquots (Table 1). Each aliquot was spiked with IA in order to obtain a final concentration of 25, 50, 100, 250, 500, 1000, or $5000 \mathrm{ng} / \mathrm{mL}$ and extracted. All quantification curves were linear between 50 and $5000 \mathrm{ng} / \mathrm{mL}$ with $R^{2}$ values ranging from 0.9951 to 0.9999 . Values for inter-assay accuracy primarily ranged from 86 to $113 \%$. Intra-assay accuracy was calculated from one aliquot of serum that was spiked with IA, extracted, and analyzed four separate times. All quantification curves were linear between 50 and $5000 \mathrm{ng} / \mathrm{mL}$ with $R^{2}$ values ranging from 0.9987 to 0.9996 . Intraassay accuracy ranges were comparable to those observed for inter-assay calculations with values also primarily ranging from 87 to $115 \%$. Precision values $(n=11)$ at the $100,250,500$, and $1000 \mathrm{ng} / \mathrm{mL}$ concentrations of 1 set of standard extracts ranged from $1.8 \%$ in the $1000 \mathrm{ng} / \mathrm{mL}$ spike to $7.7 \%$ in the $100 \mathrm{ng} / \mathrm{mL}$ spike.

IA was spiked into feline, canine, bovine, and equine serum separately in single experiments in the same manner as was done for porcine serum. All quantification curves were linear between 50 and $5000 \mathrm{ng} / \mathrm{mL}$ and the limit of detection was $25 \mathrm{ng} / \mathrm{mL}$. Further, feline (0.9914), canine (0.9913), bovine (0.9981) and equine $(0.9995)$ exhibited $R^{2}$ values closely corresponding to those observed in spiked porcine serum sample. Taken together, this precision and accuracy data suggests that (-) ESI/LC-MS is a reliable, repeatable method that may be used to identify and quantitate IA in serum.

\subsection{Field research}

In the past, IA has been successfully used as a marker in bait acceptance studies in a variety of animal species including carnivores [6-11], herbivores [12-16] and omnivores [17] and has been used to determine the feasibility of delivering oral vaccine to wild swine [17] and raccoons [9,24]. Field experiments in which feral pigs (S. scrofa), raccoons (P. lotor), and opossums (D. virginiana) were exposed to IA following ingestion of IA-marked baits were used to confirm the appropriateness of (-)ESI/LC-MS identification in these studies. Fig. 8a shows that the retention times and mass spectra of the IA peak in extracted porcine serum was comparable to that observed for bench-spiked serum samples (Fig. 7a). Similar results were observed in both raccoon and opossum serum (Figs. 9a and 10a). In addition, serum samples collected from ferrel pigs, opossums, and raccoons that did not ingest IA spiked baits failed to exhibit the characteristic peak at $\mathrm{m} / \mathrm{z} 571$ or the MS/MS peak at 443. These sera also showed no interfering peaks (Figs. 8b-10b). These results further show the effectiveness of this method for detection of IA in the serum of animals following ingestion of the compound in bait. However, while this method was shown to be appropriate for identification of IA and therefore, may be used to confirm at least partial bait ingestion, it was not intended as a means of quantification.

Although the LC-MS methodology described here allows for the direct determination of the marker following a relatively quick serum extraction that is effective for diagnostic purposes there are several factors that may still confound precise quantification. Most importantly, animals may consume only part of a bait or only part of marker within a bait, as it is difficult to achieve homogeneity within each bait, and multiple animals may consume a single bait. In addition, the extent and duration of IA binding is both species- and dose-dependent. For example, coyotes fed $0.5 \mathrm{mg} / \mathrm{kg}$ exhibited elevated iodine levels for approximately 8 weeks [6] while the duration of plasma or serum iodine elevation in stoats was only 2 weeks [21] and in the brushtail opossum and the swamp wallaby it was elevated for less than 2 weeks [11,20]. Dose-dependency has been described in beagle dogs and red foxes which, when fed $20 \mathrm{mg}$ IA, exhibited elevated iodine levels for almost twice as long as those fed $10 \mathrm{mg}$ IA [7]. Finally, many animals will not accept an unfamiliar bait (i.e., strange odor or taste) [25]. Disregarding these limitations may result in biased data regardless of the specificity and sensitivity of the analytical methodology. 


\section{References}

[1] R. Estrada, A. Vos, R. De Leon, T. Mueller, BMC Infect. Dis. 1 (2001) 23.

[2] T.J. Sidwa, P.J. Wilson, G.M. Moore, E.H. Oertli, B.N. Hicks, R.E. Rohde, D.H. Johnston, J. Am. Vet. Med. Assoc. 227 (2005) 785

[3] R.C. Rosatte, K.F. Lawson, J. Wildl. Dis. 37 (2001) 730.

[4] A. Gleixner, H.H. Meyer, A. Vos, L. Aylan, Vet. Rec. 143 (1998) 65.

[5] T.E. Creekmore, T.E. Rocke, J. Hurley, J. Wildl. Dis. 38 (2002) 32.

[6] G.E. Larson, P.J. Savarie, I. Okuno, J. Wildl. Manage. 45 (1981) 1073.

[7] G.M. Baer, J.H. Shaddock, D.J. Hayes, P. Savarie, J. Wildl. Manage. 49 (1985) 49.

[8] E.H. Follmann, P.J. Savarie, D.G. Ritter, G.M. Baer, J. Wildl. Dis. 23 (1987) 709.

[9] J. Hadidian, S.R. Jenkins, D.H. Johnson, P.T. Savarie, V.F. Nettles, D. Maiski, G.M. Baer, J. Wildl. Dis. 25 (1989) 1.

[10] G. Saunders, S. Harris, C.T. Eason, Wildl. Res. 20 (1993) 297.

[11] C.T. Eason, D. Batcheler, C.M. Frampton, Wildl. Res. 21 (1994) 377.

[12] C.T. Eason, D. Batcheler, Wildl. Res. 18 (1991) 85
[13] J.P. Parkes, Wildl. Res. 18 (1991) 687.

[14] L.M. White, D.P. Huetter, S.B. Linhart, P.J. Savarie, M.D. van Brackle, Wildl. Soc. Bull. 23 (1995) 194

[15] D.R. King, M.H. Robinson, C.T. Eason, D. Batcheler, Wildl. Res. 25 (1998) 65

[16] P.J. Sweetapple, G. Nugent, Wildl. Res. 25 (1998) 649.

[17] W.O. Fletcher, T.E. Creekmore, M.S. Smith, V.F. Nettles, J. Wildl. Dis. 26 (1990) 502.

[18] K.J. Fehske, W.E. Muller, Res. Commun. Chem. Pathol. Pharmacol. 19 (1978) 119.

[19] G.H. Mudge, N. Desbiens, G.R. Stibitz, Drug Metab. Dispos. 6 (1978) 432

[20] P.M. Fisher, C.A. Marks, Wildl. Res. 24 (1997) 97.

[21] E.B. Spurr, N. Z. J. Zool. 29 (2002) 135.

[22] A. Jones, J. Chromatogr. B 654 (1994) 293

[23] J. Blanchard, J. Chromatogr. 226 (1981) 455.

[24] S.B. Linhart, F.S. Blom, R.M. Engeman, H.L. Hill, T. Hon, D.I. Hall, J.H. Shaddock, J. Wildl. Dis. 30 (1994) 185.

[25] E.P. Benson, P.A. Zungoli, M.B. Riley, J. Econ. Entomol. 96 (2003) 94. 